\title{
Interdisziplinäre Zusammenarbeit: unabdingbare Voraussetzung für die Erforschung der Pathogenese, Diagnostik und Therapie
}

Der 118. Kongress der Deutschen Gesellschaft für Innere Medizin ist bereits „Geschichte“. Es würde mich freuen, wenn viele Leser der DMW den Kongress besucht hätten und sie mit der Auswahl der Themen und Referenten einverstanden waren. Ich erlaube mir zu wiederholen, dass sich das Gebiet der Inneren Medizin nicht als Summe ihrer acht Schwerpunkte und drei Zusatzbezeichnungen versteht. Bei der Mehrzahl der Erkrankungen innerer Organe ist zwar der Spezialist im jeweiligen Schwerpunkt der primär kompetenteste Ansprechpartner, um zum Beispiel Herzinfarkt, chronisch obstruktive Lungenerkrankung oder Leberzirrhose zu nennen, die interdisziplinäre Zusammenarbeit der Schwerpunkte ist aber oft unverzichtbar. Ich darf den Kollegen, die die Themen dieser DGIM Gesellschaftsausgabe ausgewählt haben, und den jeweiligen Autoren danken, dass es ihnen gelungen ist, diese interdisziplinäre Sichtweise darzustellen. Auch neue wissenschaftliche Erkenntnisse und die aktuelle klinische Relevanz werden in diesen Beiträgen vorzüglich dargestellt.

So ist das Bauchaortenaneurysma natürlich ein „Thema“ des Angiologen. Die Erkrankung wird aber nicht selten als Zufallsbefund vom Gastroenterologen anlässlich einer Sonografie des Abdomens diagnostiziert. Stoffwechsel/Metabolismus ist ein weiteres Beispiel der primär internistischen Sichtweise. Die wissenschaftliche Fachgesellschaft des Schwerpunkts Gastroenterologie ist die Deutsche Gesellschaft für Verdauungsund Stoffwechselkrankheiten. Niemand wird in Abrede stellen, dass der Diabetes mellitus oder die Hyperthyreose - Erkrankungen, bei denen der Endokrinologe primär angesprochen ist auch Stoffwechselkrankheiten sind. Die interdisziplinäre Diagnostik und Therapie beschränkt sich nicht nur auf das Gebiet der Inneren Medizin, wie der Beitrag aus Dresden zum Problem der Parodontitis zeigt.

Ernährung bei Demenz ist ein wirklich brennendes Problem. Wann soll der Gastroenterologe via PEG (perkutane endoskopische Gastrostomie) eine Ernährungssonde legen? Der Geriater kann sicher am kompetentesten, unter Berücksichtigung der Multimorbidität, entscheiden, wann wie zu ernähren ist. Die chronische lymphatische Leukämie ist die häufigste Leukämieform, die wiederum überwiegend bei Älteren auftritt. Bei dieser Erkrankung sind unter bestimmten Indikationen erfolgversprechendere Therapieformen als das fast schon Jahrzehnte lang angewandte „Knospe“-Schema (Chlorambucil und
Prednisolon) hinzugekommen, um nur den monoklonalen Antikörper Rituximab zu nennen, der gegen das Epitop CD-20 auf B-Lymphozyten gerichtet ist. Malaria tropica ist bezüglich der Symptomatik oft eine Chimäre. Wird bei grippalen Symptomen und fehlender Erhebung einer Reiseanamnese an diese Erkrankung nicht gedacht, kann sie tödlich verlaufen. Malaria tropica ist eine Erkrankung, die infektiologische und intensivmedizinische Expertise erfordert. Intensivmedizinische Expertise erfordert auch die Behandlung der fulminant verlaufenden akuten Pankreatitis. Bis heute suchen wir nach einer „pankreasspezifischen" Therapie. Interventionelle Radiologen und Gastroenterologen haben die Rolle des Chirurgen in der Therapie der Nekrosen etwas in den Hintergrund gedrängt. Die akute Pankreatitis ist aber ein Paradebeispiel der Notwendigkeit interdisziplinärer Diagnostik und Therapie.

Wir kommen wieder zum „Stoffwechsel“. Der Internist und seine Spezialisten wie Angiologen, Geriater, Kardiologen, aber auch Neurologen müssen aufgrund der derzeitigen Studienlage diskutieren, wann welche Zielgrößen von HDLund LDL-Cholesterin zur Primär- und Sekundärprophylaxe erreicht werden sollten. Auch der Gastroenterologe und Endokrinologe, um hier nur Adipositas, Fettleber, Gallensteine, akute Pankreatitis zu nennen, interessieren sich für den „Fettstoffwechsel“. Nierenerkrankungen sind häufig Systemerkrankungen und erfordern auch die Expertise der Rheumatologen, Endokrinologen/Diabetologen oder Kardiologen. Dem Thema Vaskulitis ist ein eigener Beitrag gewidmet. Der Pneumologe wird in der Diagnostik der „Volkskrankheit“ chronisch obstruktive Lungenerkrankung auch den Kardiologen nach dem Vorliegen einer Rechtsherzbelastung fragen.

Über Ihr Interesse an dieser Ausgabe würde ich mich daher sehr freuen.

Mit freundlichen kollegialen Grüßen Ihr

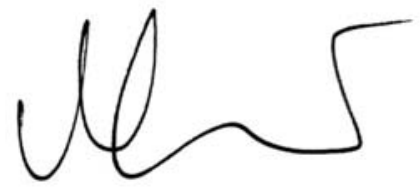

Prof. Dr. Joachim Mössner, Leipzig

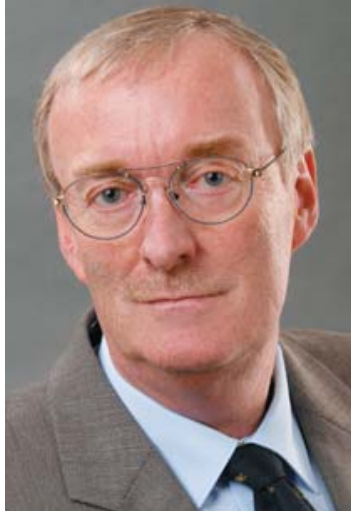

Jössner

Innere Medizin

Institut

Klinik und Poliklinik für Gastroenterologie und Rheumatologie, Department für Innere Medizin, Neurologie und Dermatologie, Universitätsklinikum Leipzig

Bibliografie

Dol $10.1055 / \mathrm{s}-0032-1305020$

Dtsch Med Wochenschr 2012;

137: 1149 - (c) Georg Thieme

Verlag KG · Stuttgart · New York - ISSN 0012-0472

Korrespondenz

Prof. Dr. med. Joachim Mössner Klinik und Polklinik für Gastroenterologie und Rheumatologie, Department für Innere Medizin, Neurologie und Dermatologie, Universitätsklinikum Leipzig, AöR Liebigstraße 20 04103 Leipzig 